\title{
How should we act on the social determinants of health?
}

\author{
Trevor J.B. Dummer PhD
}

Cite as: CMAJ 2018 October 22;190:E1241-2. doi: 10.1503/cmaj.181241

See related article at www.cmaj.ca/lookup/doi/10.1503/cmaj.180272

$\mathbf{T}$ he concept of the social determinants of health was born out of the recognition that, although disease is a biomedical outcome, socioeconomic inequities are important drivers of disease variation globally, nationally and locally..$^{1,2}$ Through a social determinants lens, it is inequities in power, money and resources that are important contributors to inequities in health, disease and mortality. ${ }^{3}$ Or more bluntly: "social injustice is killing on a grand scale." Importantly, mapping social inequities onto health variations supports upstream disease prevention and control by providing a focus for multisector interventions. However, there are limitations to the application of the concept of social determinants globally, within countries and locally.

In linked research, Wang and colleagues ${ }^{4}$ analyzed more than 40 years of data from Shanghai, China, one of the world's most populated cities. Describing variations in cause-specific mortality against a changing socioeconomic and public health landscape, they note a decreasing proportion of deaths owing to communicable diseases, injuries, and perinatal and maternal causes, combined with an increasing proportion of deaths from noncommunicable causes, including cancer and cardiovascular disease. This pattern is consistent with the epidemiological transition model, ${ }^{5}$ which conceptualizes how improvements in medicine, public health and environmental and social conditions, affect health, disease and mortality.

More specifically, Wang and colleagues described declining mortality from communicable diseases following improvements to the public health infrastructure in Shanghai during the 1980s - infrastructure developments that led to enhanced infectious disease control. ${ }^{4}$ The effect of famine in early life on increases in mortality from a range of chronic diseases is also described. Societal changes in behaviourrelated health risks, including increased tobacco smoking and alcohol consumption, physical inactivity and changes in diet leading to increasing obesity, are described in relation to increasing deaths from cancer, cardiovascular disease and other chronic conditions. All of these "risk factors" sit within the social determinants of health.

\section{KEY POINTS}

- By acknowledging the social determinants of health we recognize that, although disease is a biomedical outcome, socioeconomic inequities are important drivers of disease variation in all jurisdictions.

- More research is required on the application and interpretation of the social determinants of health in the Global South; the Global North could benefit from incorporating perspectives and insights from the Global South.

- Few studies have assessed robustly interventions that target the social determinants of health; more evaluative studies are required.

Although the concept of social determinants of health is important for describing and understanding inequities in health, globally, nationally and locally, it has limitations. I see two major challenges to acting on social determinants of health today. First, how do we apply the social determinants of health to countries outside the Global North, and, second, how should we translate evidence into policy and practice to modify or affect the social determinants of health?

In 2012, the United Nations Member States endorsed the "Rio Political Declaration on Social Determinants of Health" (Rio Declaration), a commitment to adopt a social determinants approach to reducing health inequities within and between countries. ${ }^{6}$ The declaration aims for countries to develop action plans to reduce health inequities, while acknowledging the importance of socioeconomic and political factors. However, a 2017 scoping review identified a paucity of publications on the social determinants of health from countries outside the Global North, thus limiting a global perspective and the application of the concept to the developing world. ${ }^{7}$ Spiegel and colleagues ${ }^{8}$ dissected the language of the social determinants of health, noting the dominance of a reductionist approach that focuses on risk factors related to individual living and working conditions or perhaps, at best, community influences, rather than bringing attention to structural and political processes, and 
social dynamics. ${ }^{8}$ These authors argued that much can be learned from the Global South, where there is a long history of examining the social processes that contribute to inequities in health. ${ }^{8}$ Their view is that the social determinants approach should be more than a reductionist theory that boils down determinants to classic risk factors and individual lifestyles. The authors also suggested a "social determination" approach, with an emphasis on the underlying social processes and structural forces that drive variations in the risk factors that affect health. ${ }^{7}$

Greater recognition of the social determinants of health represents a welcome shift away from a focus on individual-level influences on health - which affords primacy to genetic and clinical factors - to a more complex viewpoint that includes societal, community and structural factors. ${ }^{6}$ Consideration of these wider structural factors provides a much-needed opportunity to focus on the upstream causes of health variations (i.e., the causes of the causes), which supports preventing disease and enabling good health for all. This moves the agenda on from a narrower focus downstream (i.e., dealing with the effects of disease), where medical and health care tackle the consequences of disease and ill health. However, focusing purely upstream may neglect disadvantaged subpopulations who are the target of downstream solutions. ${ }^{6}$ Hence, it is important that, if we shift to tackling upstream social determinants, we do not lose focus of the important and successful downstream approaches.

Acting on social determinants of health and their pathways, to prevent ill health, requires more than just epidemiological data on the factors underlying these determinants. We currently lack extensive data on whether interventions aiming to modify the social determinants actually work. ${ }^{9}$ Therefore, it is important that the applied research agenda includes research that assesses interventions and evaluates the effect of policy and practice on the pathways of the social determinants of health. This is no simple task. Social determinants of health are complex; multiple diseases and causes intersect many different intervention settings, in the context of political, cultural and socioeconomic diversity. ${ }^{9}$ Although the concept of the social determinants - inequities in power, money and resources that give rise to inequities in health - may be simple, tackling these inequities is much more complex.

If we emphasize the role of structural forces and societal dynamics in the social determinants of health, we find an opportunity to tackle the causes of the causes of health inequities. But, there are challenges to overcome before they can be properly addressed.

\section{References}

1. Working Group for Monitoring Action on the Social Determinants of Health. Towards a global monitoring system for implementing the Rio Political Declaration on Social Determinants of Health: developing a core set of indicators for government action on the social determinants of health to improve health equity. Int J Equity Health 2018;17:136.

2. Krumeich A, Meershoek A. Health in global context; beyond the social determinants of health? Glob Health Action 2013;7:23506.

3. Donkin A, Goldblatt P, Allen J, et al. Global action on the social determinants of health. BMJ Glob Health 2017;3:e000603.

4. Wang S, Du X, Han X, et al. Influence of socioeconomic events on cause-specific mortality in urban Shanghai, China, from 1974 to 2015: a population-based longitudinal study. CMAJ 2018;190:E1153-61.

5. Cook IG, Dummer TJB. Changing health in China: re-evaluating the epidemiological transition model. Health Policy 2004;67:329-43.

6. Stankiewicz A, Herel M, DesMeules M. Report summary - Rio Political Declaration on Social Determinants of Health: a snapshot of Canadian actions 2015. Health Promot Chronic Dis Prev Can 2015;35:113-4.

7. Lucyk K, McLaren L. Taking stock of the social determinants of health: a scoping review. PLoS One 2017;12:e0177306.

8. Spiegel JM, Breilh J, Yassi A. Why language matters: insights and challenges in applying a social determination of health approach in a North-South collaborative research program. Global Health 2015;11:9.

9. McQueen DV. Three challenges for the social determinants of health pursuit. Int J Public Health 2009;54:1-2.

\section{Competing interests: None declared.}

This article was solicited and has not been peer reviewed.

Affiliations: Centre of Excellence in Cancer Prevention, School of Population and Public Health, The University of British Columbia, Vancouver, BC

Correspondence to: Trevor Dummer, trevor.dummer@ubc.ca 